\title{
Variants in TTC25 affect autistic trait in patients with autism spectrum disorder and general population
}

\author{
Dina Vojinovic ${ }^{1}$, Nathalie Brison ${ }^{2}$, Shahzad Ahmad ${ }^{1}$, Ilse Noens ${ }^{3,4}$, Irene Pappa ${ }^{5,6}$, Lennart C Karssen ${ }^{1,7}$, \\ Henning Tiemeier ${ }^{1,8}$, Cornelia M van Duijn ${ }^{1,9}$, Hilde Peeters ${ }^{2,3,10}$ and Najaf Amin ${ }^{\star, 1,10}$ \\ Autism spectrum disorder (ASD) is a highly heritable neurodevelopmental disorder with a complex genetic architecture. \\ To identify genetic variants underlying ASD, we performed single-variant and gene-based genome-wide association studies using \\ a dense genotyping array containing over $\mathbf{2 . 3}$ million single-nucleotide variants in a discovery sample of 160 families with at \\ least one child affected with non-syndromic ASD using a binary (ASD yes/no) phenotype and a quantitative autistic trait. \\ Replication of the top findings was performed in Psychiatric Genomics Consortium and Erasmus Rucphen Family (ERF) cohort \\ study. Significant association of quantitative autistic trait was observed with the TTC25 gene at $17 \mathrm{q} 21.2$ (effect size $=10.2$, \\ $P$-value $\left.=3.4 \times 10^{-7}\right)$ in the gene-based analysis. The gene also showed nominally significant association in the cohort-based \\ ERF study (effect $=1.75, P$-value $=0.05$ ). Meta-analysis of discovery and replication improved the association signal \\ $\left(P\right.$-value meta $\left.=1.5 \times 10^{-8}\right)$. No genome-wide significant signal was observed in the single-variant analysis of either the binary \\ ASD phenotype or the quantitative autistic trait. Our study has identified a novel gene TTC25 to be associated with quantitative \\ autistic trait in patients with ASD. The replication of association in a cohort-based study and the effect estimate suggest that \\ variants in TTC25 may also be relevant for broader ASD phenotype in the general population. TTC25 is overexpressed in frontal \\ cortex and testis and is known to be involved in cilium movement and thus an interesting candidate gene for autistic trait. \\ European Journal of Human Genetics (2017) 25, 982-987; doi:10.1038/ejhg.2017.82; published online 17 May 2017
}

\section{INTRODUCTION}

Autism spectrum disorder (ASD) is a neurodevelopmental disorder characterized by deficits in social communication and social interaction and restricted and repetitive patterns of activities and behaviour with an onset in early development. ${ }^{1}$ However ASD is a psychiatric diagnosis based on clinical criteria, and the severity of these characteristics can be measured as quantitative traits that represent a continuum that extends into the general population, with ASD at the extreme end of the distribution. ${ }^{2}$ Other associated, but not core features are intellectual disability, attention-deficit disorder and medical comorbidities. ${ }^{3}$ The prevalence of ASD is estimated to be $62 / 10000^{4}$ with boys-to-girls ratio of $\sim 4: 1 .{ }^{5}$ The importance of a genetic aetiology is established with heritability estimates ranging from 37 to $90 \%{ }^{6-9}$ Despite genetic heterogeneity, considerable progress in understanding the genetic architecture of ASD has been made by identifying monogenetic causes through genetic syndromes, ${ }^{10}$ rare chromosomal abnormalities, ${ }^{11,12}$ rare copy-number variants ${ }^{13-16}$ and rare penetrant gene mutations. ${ }^{3}$ Several genomic regions, including $2 \mathrm{q}$, $3 \mathrm{q} 25-27,3 \mathrm{p} 25,6 \mathrm{q} 14-21,7 \mathrm{q} 31-36$ and $17 \mathrm{q} 11-21^{17}$ have been linked to ASD. The role of rare genetic variants in the aetiology of ASD has been established by high-throughput technologies. ${ }^{18,19}$ More recently, the theory of excess of de novo loss-of-function variants in ASD patients has gained popularity after some initial successes. ${ }^{18-20}$ Around
1000 genes have been identified to be enriched with de novo loss-offunction mutations in ASD patients. ${ }^{21}$ However, de novo genetic variants do not contribute to the estimated heritability as these are not inherited. On the other hand, most genetic variance in ASD is attributed to common genetic variants. ${ }^{9,22}$ Their role has been demonstrated by several genome-wide association studies $\left(\right.$ GWAS) ${ }^{23-29}$ (Supplementary Table S1). Even though not many common susceptibility variants have been identified, significant association has been reported at $5 \mathrm{p} 14.1,1^{23}$ at $5 \mathrm{p} 15.31$ between SEMA5A and TAS2R1 genes, ${ }^{28}$ within MACROD2 at 20p12.126 and at 1 p13.2. ${ }^{29}$ However, there is a significant overlap of the discovery samples used and little replication of specific loci between studies. ${ }^{30}$

Although the individual effect of common variants is modest, their joint effect may be substantial. ${ }^{25}$ In this study besides assessing the effect of single variants on ASD, we evaluated the joint effect of multiple single variants in a gene in a genome-wide gene-based association analysis in patients with ASD from a Belgian Flemish cohort who were genotyped on a dense genotyping array.

\section{MATERIALS AND METHODS}

General overview of the study design and work flow are illustrated in Supplementary Figure S1.

${ }^{1}$ Department of Epidemiology, Erasmus University Medical Center, Rotterdam, The Netherlands; ${ }^{2}$ Center for Human Genetics, University Hospitals Leuven, KU Leuven, Leuven, Belgium; ${ }^{3}$ Leuven Autism Research (LAuRes), Leuven, Belgium; ${ }^{4}$ Parenting and Special Education Research Unit, KU Leuven, Leuven, Belgium; ${ }^{5}$ School of Pedagogical and Educational Sciences, Erasmus University Rotterdam, Rotterdam, The Netherlands; ${ }^{6}$ Generation R Study Group, Erasmus University Medical Center, Rotterdam, The Netherlands; ${ }^{7}$ PolyOmica, s-Hertogenbosch, The Netherlands; ${ }^{8}$ Department of Child and Adolescent Psychiatry/Psychology, Erasmus University Medical Center-Sophia Children's Hospital, The Netherlands; ${ }^{9}$ Leiden Academic Centre for Drug Research (LACDR), Leiden University, The Netherlands

*Correspondence: Dr N Amin, Department of Epidemiology, Erasmus University Medical Center, PO Box 2040, Rotterdam 3000 CA, The Netherlands. Tel +31 107038125 ; Fax: +31 1070446 57; E-mail: n.amin@erasmusmc.nl

10These authors contributed equally to this work.

Received 29 August 2016; revised 3 April 2017; accepted 13 April 2017; published online 17 May 2017 


\section{Discovery sample}

The discovery sample consisted of 160 nuclear Belgian Flemish families (657 individuals; Supplementary Table S2). The families were recruited to participate in the prospective study through the Expert Center for Autism (ECA) Leuven. All probands had been seen multiple times as part of their clinical care program in the ECA before recruitment. The families were asked to participate if there was at least one child with the diagnosis of non-syndromic ASD of unknown origin after a clinical genetics workup. Out of the 160 families, 55 were multiplex (two or more siblings with ASD) and 105 simplex. In six families the father had also been diagnosed with ASD such that there were $77.7 \%$ affected males and $22.3 \%$ affected females with male-to-female ratio of 3.5:1. Among them, $88.4 \%$ had normal and high intelligence, whereas $11.6 \%$ had mild, moderate or severe intellectual disability.

Diagnoses of ASD were made by a multidisciplinary team in the ECA Leuven according to DSM-IV-TR (American Psychiatric Association, 2000) criteria. Additionally, participants were assessed for quantitative autistic trait using the Dutch version of the Social Responsiveness Scale (SRS) and the Social Responsiveness Scale for Adults (SRS-A) designed to measure social impairment associated with ASD across a wide range of severity. ${ }^{31,32}$ Completed questionnaires were obtained for 490 probands, parents and siblings. Among the affected patients that had the SRS score available, the majority had normal and high intelligence (86\%). For all participants, we received written informed consent. This study was approved by the Medical Ethical Committee of the University Hospitals Leuven.

\section{Genotyping}

Genotyping of 657 individuals from the discovery cohort was performed at the Center for Human Genetics at the KU Leuven, Belgium using the HumanOmni2.5-8 BeadChip, which contains more than 2.3 million common and lessfrequent single-nucleotide polymorphisms (SNPs) from the 1000 Genome Project (minor allele frequency $>2.5 \%$ ). SNP calling was performed in Genome Studio 2011.1 using the genotyping module v1.9. Markers with call rate $<95 \%$, or which were monomorphic or which failed an exact test of Hardy-Weinberg equilibrium (HWE) $\left(P\right.$-value $\left.<1 \times 10^{-7}\right)$ were removed from the analysis. Samples with low call rate $<95 \%$ or high identity-by-state ( $295 \%)$ were also removed from the analysis. Ethnic outliers were determined using multidimensional scaling analysis with 1000 Genomes data set (Supplementary Figure S2). All samples clustered tightly with the Europeans and no ethnic outlier was identified. In total 1646898 markers and 654 genotyped individuals were retained for further statistical analysis. Lastly, Mega2 tool v4.4(ref. 33) was used to identify Mendelian inconsistencies, which were later set to missing.

\section{Statistical analysis}

Baseline descriptive analysis was performed with SPSS v21 (IBM Corporation, Armonk, NY, USA) and PEDSTATS v0.6.12. ${ }^{34}$ Genome-wide association analyses of the binary ASD phenotype were performed through joint modelling of linkage and association, using the LAMP software v0.0.9 (School of Public Health, Ann Arbor, MI, USA). LAMP uses a maximum likelihood model to extract information on genetic linkage and association from samples of unrelated individuals, sib pairs, trios and larger pedigrees in settings where population stratification is not a concern (Supplementary Figure S2). ${ }^{35}$ Odds ratios and 95\% confidence intervals were estimated using PLINK v1.07. ${ }^{36}$ The association tests for markers on sex chromosomes were performed by transmission disequilibrium test for chromosome $\mathrm{X}$ and by logistic regression for chromosome Y. Single-variant and gene-based genome-wide association analyses of the quantitative autistic trait adjusted for age, gender and familial relationships were performed using the RVtests software tool version 20150630 (http://zhanxw.github.io/ rvtests/). The gene-based analysis included Combined Multivariate and Collapsing method which is robust and powerful in the presence of wide spectrum of variant allele frequencies. ${ }^{37}$ The genes were defined according to human reference genome hg19. All association analyses were performed for entire discovery sample, and simplex and multiplex families separately. The standard genome-wide significance threshold of $5 \times 10^{-8}$ was used to declare significance in the single-variant analyses, while the genomewide significance threshold for the gene-based analysis was set at $2.5 \times 10^{-6}$ based on 19650 genes tested. PLINK/SEQ v0.10 (https://atgu.mgh.harvard.edu/ plinkseq/) was used to convert PLINK files into variant call format files. All genome maps were updated to human genome build 19 (hg19). Gene pathway enrichment analysis of all nominally significant genes $(P$-value $<0.01)$ in the gene-based analysis was performed using the web-based gene network pathway enrichment tool (http://129.125.135.180:8080/GeneNetwork/pathway.html).

The data were deposited in the GWAS Central database (http://www. gwascentral.org/study/HGVST1847).

\section{Bioinformatic analysis}

To annotate SNPs with regulatory information, we used RegulomeDB v1.1 database (http://www.regulomedb.org/index) that combines information from ENCODE and other sources, as well as computational predictions and manual annotations into a tool that classifies SNPs into six categories, where Category 1 variants are 'likely to affect binding and linked to expression of a gene target', whereas category 6 variants have 'minimal binding evidence'. ${ }^{38}$ Furthermore, regulatory information on SNPs in haplotype blocks was explored using a HaploReg v4.1 tool..$^{39}$ For these analysis $r^{2}$ was set to 1 and the population of European descent was chosen.

\section{Replication samples}

Psychiatric Genomics Consortium (PGC). A lookup of top findings from the single-variant analyses of the binary ASD phenotype and quantitative autistic trait was performed in the latest PGC GWAS. This data set consists of a total of 6495 parent-child trios who met diagnostic criteria for ASD and had genomewide SNP data available (https://www.med.unc.edu/pgc).

Erasmus Rucphen Family (ERF) study. Replication of the gene-based analysis of quantitative autistic trait was performed in the ERF study as 1250 participants from this cohort have been assessed for quantitative autistic trait using Baron-Cohen's Autism-Spectrum Quotient (AQ) test ${ }^{40}$ and exomes of half of these participants $(n=615)$ have been sequenced, thus providing a greater resolution at the gene level. Individuals whose exome were sequenced were selected based on having good quality phenotype information on a wide range of topics, and therefore random with regards to AQ scores (Supplementary Table S2). ERF is a family-based cohort originating from 22 couples and spread over 23 generations. ${ }^{41,42}$ The ERF study was approved by the Medical Ethics Committee of the Erasmus MC which is constituted according to the WMO (Wet Medisch-wetenschappelijk Onderzoek met mensen). A written informed consent was obtained from all study participants.

Sequencing was done at a mean depth of $74 \times$ using the Nimblegen SeqCap EZ V2 capture kit on an Illumina Hiseq2000 sequencer (Illumina, San Diego, CA, USA) using the TruSeq Version 3 protocol at the Human Genotyping facility of the Internal Medicine department, at the Erasmus MC, The Netherlands. ${ }^{43,44}$ The sequence reads were aligned to the human genome build 19 (hg19), using Burrows-Wheeler Aligner and the NARWHAL pipeline. ${ }^{45,46}$ After processing, genetic variants were called, using the Unified Genotyper tool from the GATK. ${ }^{47}$ Variants with a low quality $(\mathrm{QUAL}<150)$, which were out of HWE $\left(P\right.$-value $\left.<10^{-6}\right)$ or with low call rate $(<90 \%)$, as well as samples with a low call rate $(<90 \%)$, and duplicates, were removed. ${ }^{44}$ Functional annotations were also performed using the SeattleSeq annotation 138 database (http://snp.gs.washington.edu/SeattleSeqAnnotation138/).

Association analyses of the quantitative autistic trait adjusted for age, gender and familial relationship were performed using the RVtests software. Metaanalysis of gene-based results of discovery sample and ERF study was performed using Fisher's combined probability test.

\section{RESULTS}

Results of genome-wide association analysis for the binary ASD phenotype and quantitative autistic trait are illustrated in Supplementary Figures S3 and S4. No single-variant surpassed the genome-wide significance threshold. Top findings from the association analyses are shown in Tables 1 and 2 for the discovery sample, and in Supplementary Tables S3 and S4 for simplex and multiplex families 
Table 1 Top findings $\left(P\right.$-value $<1 \times 10^{-5}$ ) from the binary ASD phenotype association analysis

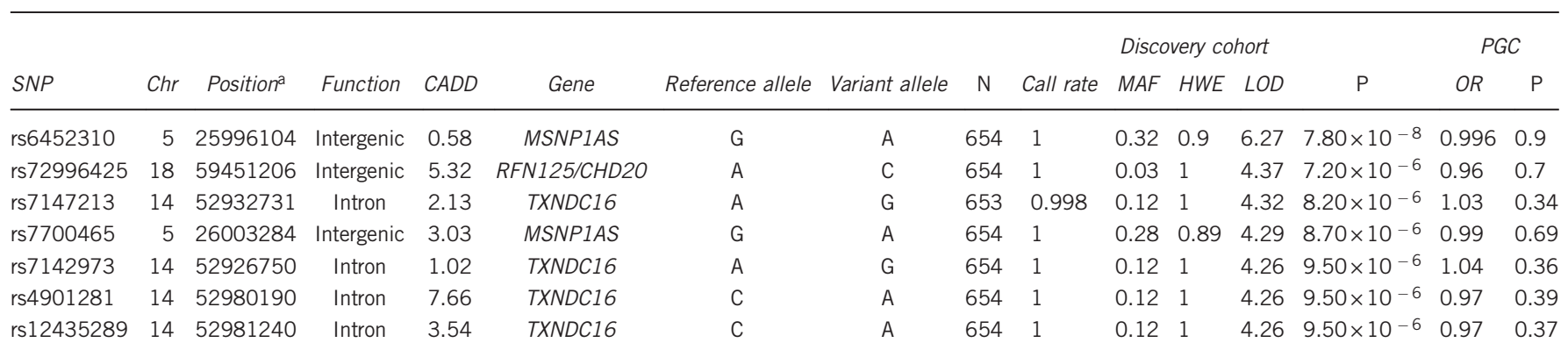

Abbreviations: ASD, autism spectrum disorder; Chr, chromosome; CADD, phred-like Combined Annotation Dependent Depletion scores from Kircher et al, ${ }^{70}$ University of Washington; HWE, HardyWeinberg equilibrium in founders; MAF, minor allele frequency; $N$, number of individuals; OR, odds ratio; PGC, Psychiatric Genomics Consortium.

a Genomic position is according the hg19 assembly.

separately. Suggestive association of binary ASD phenotype was observed with two common variants (rs6452310; $P$-value $=7.80 \times 10^{-8}$ and rs7700465; $P$-value $=8.70 \times 10^{-6}$; Table 1$)$ at chromosome 5 p14.1-a region previously known to be associated with ASD (Figure 1). The two variants were in strong linkage disequilibrium (LD) $\left(r^{2}=0.85\right)$ with each other but not in LD with any of the previously identified variants in this region associated with $\mathrm{ASD}^{23,24}$ ( $r^{2}$ ranged from 0.002 to $0.009, D^{\prime}$ ranged from 0.02 to 0.33 ). None of the top variants from this analysis showed evidence of association in the replication sample (Table 1).

Results of gene-based genome-wide association analysis are illustrated in Supplementary Figure S5 for the discovery sample, and in Supplementary Table S5 for simplex and multiplex families separately. The gene-based association analysis revealed significant association of quantitative autistic trait and TTC25 gene $\left(P\right.$-value $\left.=3.4 \times 10^{-7}\right)$ on chromosome 17 (Table 3 ). This association was not driven by any single variant but by nine variants, four of which showed nominally significant association with quantitative autistic trait $(P$-value $<0.05)$ (Supplementary Table S6). The combined effect of these variants on the SRS score was large (effect size $=10.2$ ). The functional annotation of nine variants revealed that five variants are likely to have regulatory functions (Category 1 RegulomeDB score; Supplementary Table S6). Furthermore, the surrounding variants in strong LD $\left(r^{2}=1\right)$ with nine variants lie in enhancer histone marks, and protein-binding regions and change regulatory motifs based on the variant allele changes (Supplementary Table S7). The gene was also nominally associated with autistic trait in the ERF cohort $(P$-value $=0.045)$. The combined effect of 15 variants in the TTC25 gene (Supplementary Table S8) in the replication sample was much smaller (effect size $=1.75$ ). Metaanalysis of discovery and replication samples resulted in an improved association signal $\left(P\right.$-value $\left.=1.5 \times 10^{-8}\right)$.

\section{Pathway analysis}

Pathway enrichment analysis based on nominally significant genes in the gene-based association analysis (Supplementary Table S9) showed significantly enriched SMAD protein signal transduction $(P$-value $=$ $2 \times 10^{-5}$ ) pathway and series of digestive system development enrichment categories (Supplementary Table S10).

\section{DISCUSSION}

In this study, we have identified a novel gene TTC25 associated with quantitative autistic trait. The association of the gene TTC25 in a cohort-based study suggests that the gene may be relevant for broader ASD phenotype in the general population. Further, we identified
SMAD protein signal transduction pathway and series of digestive system development categories as being significantly enriched with genes nominally associated with quantitative autistic trait. Moreover, our study provides additional evidence for the previously identified association of the intergenic loci at 5p14.1 with the binary ASD phenotype.

TTC25 gene is located on chromosome 17q21.2 and encodes Tetratricopeptide Repeat Domain 25. 17q21.2 locus has previously been linked to ASD in a genome-wide linkage scan, ${ }^{48,49}$ although a gene was never implicated. TTC25 is overexpressed in testis, frontal cortex and rectum (http://www.genecards.org/cgi-bin/carddisp.pl? gene $=$ TTC25). TTC25 is involved in cilium movement, organization and morphogenesis. ${ }^{50}$ Cilia are specialized organelles protruding from the cell surface of almost all mammalian cells. ${ }^{51}$ Mutations in ciliary proteins cause ciliopathies which can affect many organs at different levels of severity and are characterized by a wide spectrum of phenotypes. ${ }^{51}$ In the vertebrate nervous system, the primary cilium is increasingly viewed as hub for certain neural developmental signalling pathways, and growing data suggest this is also true for several types of adult neuronal signalling. ${ }^{52}$ The capacity of the brain to interpret the sensory input is often affected in ciliopathies, resulting in neurological disorders; cognitive impairment, anosmia, intellectual disability, ASD and obesity are apparent in various degrees in many of the ciliopathies. ${ }^{51,53}$ Further Joubert syndrome (JS) is a well-known ciliopathy of the central nervous system. ${ }^{52,54}$ Features of ASD, such as problems in social behaviour, communication problems and repetitive behaviours, have been described in up to $40 \%$ of JS patients $5^{5-57}$ and about $25 \%$ of JS patients meet criteria for the DSM-IV diagnosis of ASD. ${ }^{55,58}$ Multiple variants mapped to this gene in our sample appear to have a regulatory function.

Our identification of SMAD protein signal transduction pathway as being significantly enriched with genes nominally associated with quantitative autistic trait reinforces the role of the transforming growth factor- $\beta$ (TGF $\beta$ ) in ASD. The Smad pathways are the major mediators of transcriptional responses induced by the TGF $\beta$ family, which control cell-fate determination, cell cycle arrest, apoptosis and actin rearrangements. ${ }^{59}$ While decreased levels of TGF $\beta$ have been reported in blood samples from individuals with $\mathrm{ASD}^{60}$ and associated with more severe behavioural scores in ASD children, ${ }^{61}$ higher levels of TGF $\beta$ have been reported in postmortem brain and cerebrospinal fluid samples of ASD patients. ${ }^{62}$ In addition, series of digestive system development categories were enriched with genes nominally associated with ASD. Gastrointestinal (GI) disturbances are 4-fold more common in $\mathrm{ASD}^{63}$ and available scientific evidence supports 


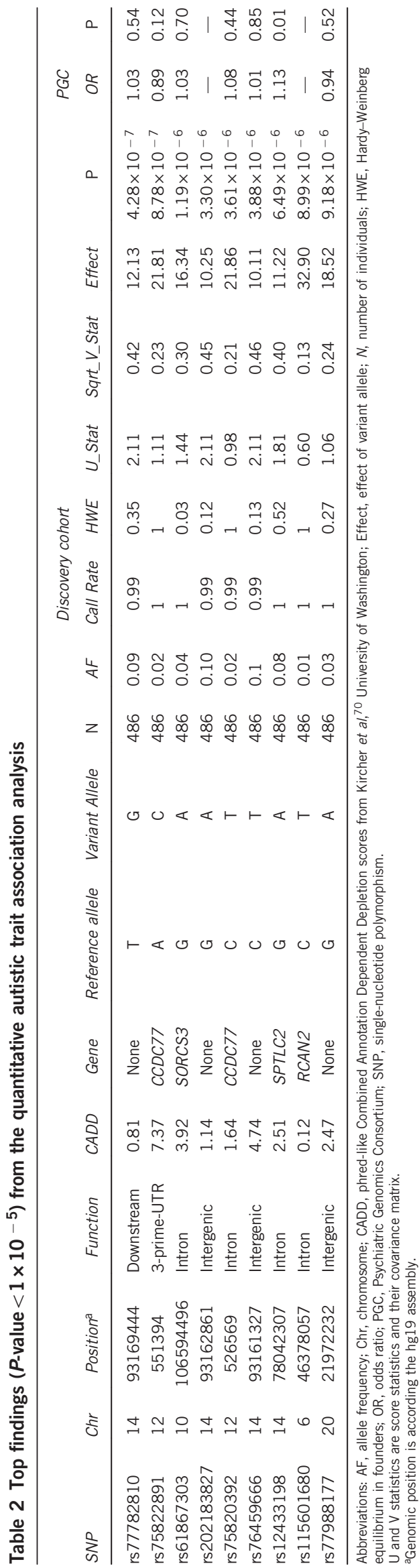

combination of changes in the areas of immune function, gut microbiome and gut and brain signalling pathways. ${ }^{64}$ Recent studies in animal models suggests that GI difficulties may originate from the same genetic changes that lead to the behavioural characteristics of ASD. 65

In addition, we found the association of ASD with the known region on 5 p14.1. This is one of the few replicated GWA regions that implicates long noncoding RNA gene, MSNP1AS (moesin pseudogene 1 , antisense) in ASD risk. ${ }^{66}$ This region has also been associated with social communication spectrum phenotypes in the general population supporting the role of 5 p14.1 as a quantitative trait locus for ASD. ${ }^{49}$ MSNP1AS shows a very high sequence homology to the chromosome $\mathrm{X}$ transcript of MSN that is involved in brain development. ${ }^{66}$ MSNP1AS is highly overexpressed (12.7-fold) in the postmortem cerebral cortex of individuals with ASD. ${ }^{66}$ Interestingly, our top hit did not replicate in the PGC sample and vice versa. The multiple different variants discovered in the 5 p14.1 region ${ }^{23,24}$ may suggest that multiple alleles in the same region are implicated in ASD.

Although our study sample was small, the strength of our study is that we used a data set with high-quality phenotypes, in which participants were assessed for both binary ASD phenotype and a quantitative autistic trait. The use of quantitative endophenotype provides additional power to find genetic signals by focusing on less complex aspects of complex phenotypes such as ASD. ${ }^{67}$ The identification of new loci associated with quantitative autistic trait in our study validates this approach. Another strength is phenotypic homogeneity in the sense that the majority of patients in the current study had a normal intelligence, unlike most ASD cohorts with typical rates of intellectual disability ranging from 30 to $50 \%{ }^{68}$ Further, the genomes of our study participants were genotyped on a very dense SNP array that contains not only common but also less-frequent SNPs. This gave us the opportunity to make a comprehensive overview of how common and less-frequent variants, both individually and taken together, affect ASD. Our study shows the advantage of a genebased test as the more powerful approach compared to single-variant analysis and demonstrated the use of gene-based pathway and enrichment analysis in understanding the molecular mechanisms of the disorder. One of the possible limitations of our study is that the assessment of quantitative autistic trait in the discovery and replication cohort used two different questionnaires, the SRS and the AQ. As the questionnaires have both been designed to measure the severity of social responsiveness problems across clinical cases and the general population and as their ratings are significantly correlated, ${ }^{69}$ we were able to compare results from the two cohorts.

To conclude, our study has identified a novel gene TTC25 to be associated with autistic trait in the ASD population where majority of patients have a normal intelligence. The replication of TTC25 association in a cohort-based study suggests that this gene may also be relevant for broader ASD phenotype in the general population. However, whether these findings hold true also for ASD patients with intellectual disability remains to be evaluated. TTC25 is overexpressed in frontal cortex and testis and is known to be involved in cilium movement and thus an interesting candidate gene for autistic trait. Furthermore, we discovered significantly enriched SMAD protein signal transduction pathway and series of digestive system development categories in the pathway analysis of quantitative autistic trait. Our finding provides new insights into the genetic background of quantitative autistic trait. 


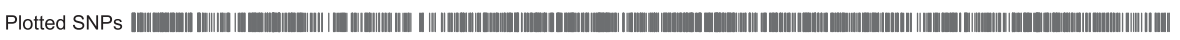

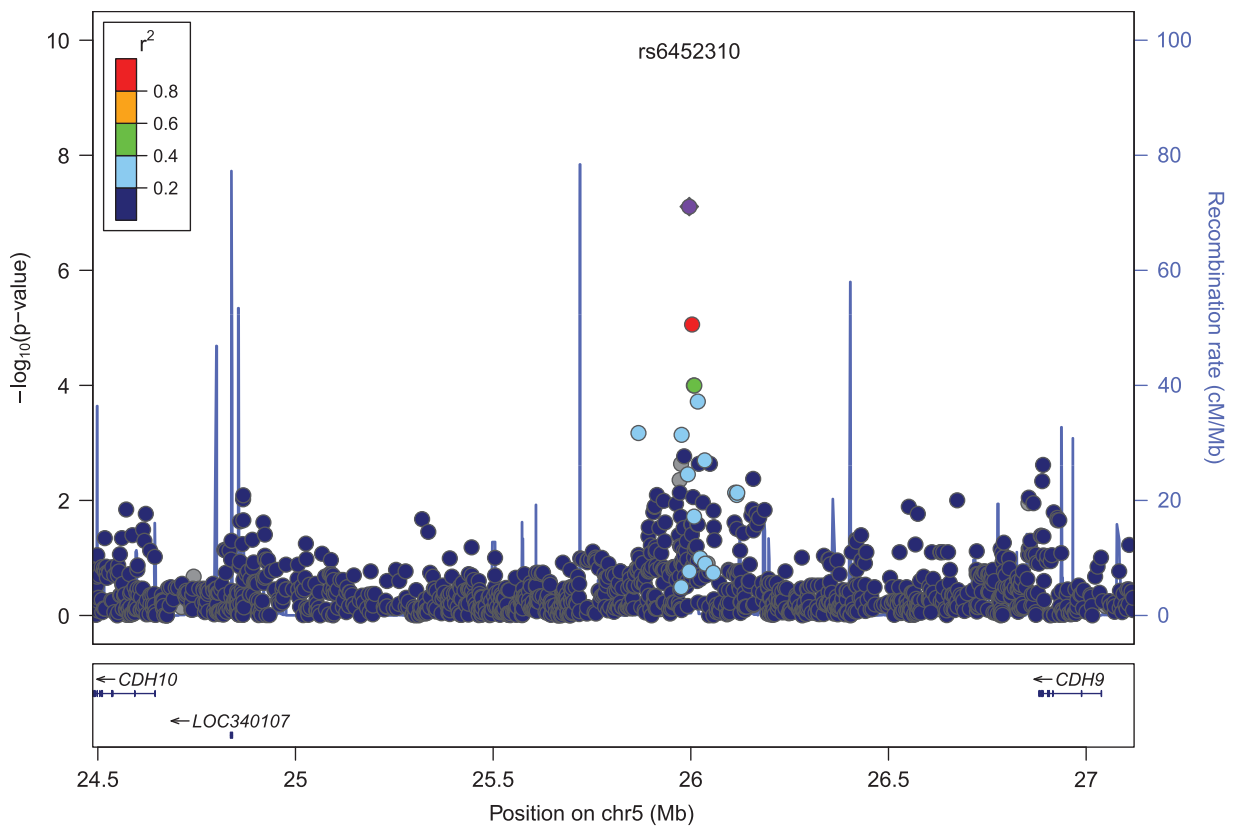

Figure 1 Regional association and recombination rate plot of the $5 \mathrm{p} 14.1$ region and the binary ASD phenotype in the discovery cohort. The left $y$ axis represents $-\log 10 P$-values for association with binary ASD phenotype in the discovery cohort. The right $y$ axis represents the recombination rate, and the $x$ axis represents chromosomal position (genomic position is according to the hg19 assembly). The most significantly associated single SNP in this region (rs6452310) is denoted with a purple diamond. Surrounding SNPs are shaded according to their pairwise correlation ( $r 2$ ) with rs6452310. The gene annotations are shown below the figure.

Table 3 Top results $\left(P\right.$-value $<1 \times 10^{-3}$ ) from the gene-based association analysis of quantitative autistic trait in the discovery cohort

\begin{tabular}{lrrrrrrr}
\hline Gene & Chr & N & NumVar & NumSite & AFa & Effect & P \\
\hline TTC25 & 17 & 486 & 13 & 9 & 0.338 & 10.23 & $3.44 \times 10^{-7}$ \\
SH2D5 & 1 & 486 & 9 & 5 & 0.440 & -10.88 & $1.49 \times 10^{-4}$ \\
PSMC5 & 17 & 486 & 4 & 3 & 0.458 & -11.13 & $6.81 \times 10^{-4}$ \\
LOC729177 & 6 & 486 & 22 & 14 & 0.434 & -9.15 & $9.37 \times 10^{-4}$ \\
\hline
\end{tabular}

Abbreviations: AF, allele frequency; Chr, chromosome; Effect, effect size; $N$, sample size; NumVar, number of variants; NumSite, number of sites.

aAllele frequency is calculated as an average of individual allele frequency adjusted for relatedness (http://zhanxw.github.io/rvtests/).

\section{CONFLICT OF INTEREST}

IN receives royalties from Hogrefe Uitgeverij for the SRS-A (although not for the questionnaires used in this study). The remaining authors declare no conflict of interest.

\section{ACKNOWLEDGEMENTS}

The Erasmus Rucphen Family study (ERF) study as a part of EUROSPAN (European Special Populations Research Network) was supported by European Commission FP6 STRP grant number 018947 (LSHG-CT-2006-01947) and also received funding from the European Community's Seventh Framework Programme (FP7/2007-2013)/grant agreement HEALTH-F4-2007-201413 by the European Commission under the programme 'Quality of Life and Management of the Living Resources' of 5th Framework Programme (no. QLG2-CT-2002-01254). High-throughput analysis of the ERF data was supported by joint grant from Netherlands Organization for Scientific Research and the Russian Foundation for Basic Research (NWO-RFBR 047.017.043). Exome sequencing analysis in ERF was supported by the ZonMw grant (project 91111025). We are grateful to all study participants and their relatives, general practitioners and neurologists for their contributions and to $\mathrm{P}$ Veraart for her help in genealogy, J Vergeer for the supervision of the laboratory work and P Snijders for his help in the data collection. NA is supported by The Netherlands Brain Foundation (project number F2013(1)-28). HP is a Senior Clinical Investigator of The Research Foundation-Flanders (FWO). We acknowledge PGC consortium for providing the pre-publication results.

1 Association AP Diagnostic and Statistical Manual of Mental Disorders. 5th edn Arlington, VA: American Psychiatric Publishing, 2013.

2 Constantino JN, Todd RD: Autistic traits in the general population: a twin study. Arch Gen Psychiatry 2003; 60: 524-530.

3 Devlin B, Scherer SW: Genetic architecture in autism spectrum disorder. Curr Opin Genet Dev 2012; 22: 229-237.

4 Elsabbagh M, Divan G, Koh YJ et al: Global prevalence of autism and other pervasive developmental disorders. Autism Res 2012; 5: 160-179.

5 Werling DM, Geschwind DH: Sex differences in autism spectrum disorders. Curr Opin Neurol 2013; 26: 146-153.

6 Hallmayer J, Cleveland S, Torres A et al: Genetic heritability and shared environmental factors among twin pairs with autism. Arch Gen Psychiatry 2011; 68: 1095-1102.

7 Bailey A, Le Couteur A, Gottesman I et al: Autism as a strongly genetic disorder: evidence from a British twin study. Psychol Med 1995; 25: 63-77.

8 Sandin S, Lichtenstein P, Kuja-Halkola R, Larsson H, Hultman CM, Reichenberg A: The familial risk of autism. JAMA 2014; 311: 1770-1777.

9 Gauglerl T, Klei L, Sanders SJ et al: Most genetic risk for autism resides with common variation. Nat Genet 2014; 46: 881-885.

10 Betancur C: Etiological heterogeneity in autism spectrum disorders: more than 100 genetic and genomic disorders and still counting. Brain Res 2011; 1380: 42-77.

11 Marshall CR, Noor A, Vincent JB et al: Structural variation of chromosomes in autism spectrum disorder. Am J Hum Genet 2008; 82: 477-488.

12 Shen Y, Dies KA, Holm IA et al: Clinical genetic testing for patients with autism spectrum disorders. Pediatrics 2010; 125: e727-e735.

13 Pinto D, Pagnamenta AT, Klei L et al: Functional impact of global rare copy number variation in autism spectrum disorders. Nature 2010; 466: 368-372.

14 Gai X, Xie HM, Perin JC et al: Rare structural variation of synapse and neurotransmission genes in autism. Mol Psychiatry 2012; 17: 402-411.

15 Sebat J, Lakshmi B, Malhotra D et al: Strong association of de novo copy number mutations with autism. Science 2007; 316: 445-449.

16 Girirajan S, Brkanac Z, Coe BP et al: Relative burden of large CNVs on a range of neurodevelopmental phenotypes. PLOS Genet 2011; 7: e1002334. 
17 Freitag CM: The genetics of autistic disorders and its clinical relevance: a review of the literature. Mol Psychiatry 2007; 12: 2-22.

18 Sanders SJ, Murtha MT, Gupta AR et al: De novo mutations revealed by whole-exome sequencing are strongly associated with autism. Nature 2012; 485: 237-241.

19 O'Roak BJ, Vives L, Girirajan S et al: Sporadic autism exomes reveal a highly interconnected protein network of de novo mutations. Nature 2012; 485: 246-250.

20 O'Roak BJ, Deriziotis P, Lee $\mathrm{C}$ et al: Exome sequencing in sporadic autism spectrum disorders identifies severe de novo mutations. Nat Genet 2011; 43: 585-589.

21 Samocha KE, Robinson EB, Sanders SJ et al: A framework for the interpretation of de novo mutation in human disease. Nat Genet 2014; 46: 944-950.

22 Cross-Disorder Group of the Psychiatric Genomics C: Identification of risk loci with shared effects on five major psychiatric disorders: a genome-wide analysis. Lancet 2013; 381: 1371-1379.

23 Wang $\mathrm{K}$, Zhang $\mathrm{H}$, Ma D et al: Common genetic variants on $5 \mathrm{p} 14.1$ associate with autism spectrum disorders. Nature 2009; 459: 528-533.

24 Ma D, Salyakina D, Jaworski JM et al: A genome-wide association study of autism reveals a common novel risk locus at 5p14.1. Ann Hum Genet 2009; 73: 263-273.

25 Anney R, Klei L, Pinto D et al: Individual common variants exert weak effects on the risk for autism spectrum disorderspi. Hum Mol Genet 2012; 21: 4781-4792.

26 Anney R, Klei L, Pinto D et al: A genome-wide scan for common alleles affecting risk for autism. Hum Mol Genet 2010; 19: 4072-4082.

27 Connolly JJ, Glessner JT, Hakonarson H: A genome-wide association study of autism incorporating autism diagnostic interview-revised, autism diagnostic observation schedule, and social responsiveness scale. Child Dev 2013; 84: 17-33.

28 Weiss LA, Arking DE, Daly MJ, Chakravarti A: Gene Discovery Project of Johns $\mathrm{H}$ and the Autism C: A genome-wide linkage and association scan reveals novel loci for autism. Nature 2009; 461: 802-808.

29 Xia K, Guo H, Hu Z et al: Common genetic variants on 1 p13.2 associate with risk of autism. Mol Psychiatry 2014; 19: 1212-1219.

30 Holt R, Monaco AP: Links between genetics and pathophysiology in the autism spectrum disorders. EMBO Mol Med 2011; 3: 438-450.

31 Roeyers H, Thys M, Druart C, De Schryver M, Schittekatte M: SRS Screeningslijst Voor Autismespectrumstoornissen. Amsterdam: Hogrefe Uitgevers B.V, 2011.

32 Noens I, De la Marche W, Scholte E: SRS Screeningslijst Voor Autismespectrumstoornissen bij Volwassenen. Amsterdam: Hogrefe Uitgevers BV, 2012.

33 Mukhopadhyay N, Almasy L, Schroeder M, Mulvihill WP, Weeks DE: Mega2: datahandling for facilitating genetic linkage and association analyses. Bioinformatics 2005; 21: 2556-2557.

34 Wigginton JE, Abecasis GR: PEDSTATS: descriptive statistics, graphics and quality assessment for gene mapping data. Bioinformatics 2005; 21: 3445-3447.

35 Li M, Boehnke M, Abecasis GR: Joint modeling of linkage and association: identifying SNPs responsible for a linkage signal. Am J Hum Genet 2005; 76: 934-949.

36 Purcell S, Neale B, Todd-Brown K et al: PLINK: a tool set for whole-genome association and population-based linkage analyses. Am J Hum Genet 2007; 81: 559-575.

$37 \mathrm{Li} \mathrm{B}$, Leal SM: Methods for detecting associations with rare variants for common diseases: application to analysis of sequence data. Am J Hum Genet 2008; 83 311-321.

38 Boyle AP, Hong EL, Hariharan M et al: Annotation of functional variation in personal genomes using RegulomeDB. Genome Res 2012; 22: 1790-1797.

39 Ward LD, Kellis M: HaploReg: a resource for exploring chromatin states, conservation, and regulatory motif alterations within sets of genetically linked variants. Nucleic Acids Res 2012; 40: D930-D934.

40 Baron-Cohen S, Wheelwright S, Skinner R, Martin J, Clubley E: The autism-spectrum quotient (AQ): evidence from Asperger syndrome/high-functioning autism, males and females, scientists and mathematicians. J Autism Dev Disord 2001; 31: 5-17.

41 Aulchenko YS, Heutink P, Mackay I et al: Linkage disequilibrium in young genetically isolated Dutch population. Eur J Hum Genet 2004; 12: 527-534.

42 Pardo LM, MacKay I, Oostra B, van Duijn CM, Aulchenko YS: The effect of genetic drift in a young genetically isolated population. Ann Hum Genet 2005; 69: 288-295.

43 Vojinovic D, Adams HH, van der Lee SJ et al: The dystrophin gene and cognitive function in the general population. Eur J Hum Genet 2015; 23: 837-843.

44 Amin N, Jovanova O, Adams HH et al: Exome-sequencing in a large population-based study reveals a rare Asn396Ser variant in the LIPG gene associated with depressive symptoms. Mol Psychiatry 2016; 22: 634.
45 Brouwer RW, van den Hout MC, Grosveld FG, van Ijcken WF: NARWHAL, a primary analysis pipeline for NGS data. Bioinformatics 2012; 28: 284-285.

46 Bucan M, Abrahams BS, Wang $\mathrm{K}$ et al: Genome-wide analyses of exonic copy number variants in a family-based study point to novel autism susceptibility genes. PLoS Genet 2009; 5: e1000536.

47 McKenna A, Hanna M, Banks E et al: The Genome Analysis Toolkit: a MapReduce framework for analyzing next-generation DNA sequencing data. Genome Res 2010; 20 1297-1303.

48 McCauley JL, Li C, Jiang L et al: Genome-wide and ordered-subset linkage analyses provide support for autism loci on $17 q$ and $19 p$ with evidence of phenotypic and interlocus genetic correlates. BMC Med Genet 2005; 6: 1.

49 Lowe JK, Werling DM, Constantino JN, Cantor RM, Geschwind DH: Social responsiveness, an autism endophenotype: genomewide significant linkage to two regions on chromosome 8. Am J Psychiatry 2015; 172: 266-275.

$50 \mathrm{Xu} \mathrm{Y,} \mathrm{Cao} \mathrm{J,} \mathrm{Huang} \mathrm{S} \mathrm{et} \mathrm{al:} \mathrm{Characterization} \mathrm{of} \mathrm{tetratricopeptide} \mathrm{repeat-}$ containing proteins critical for cilia formation and function. PLoS One 2015; 10 e0124378.

51 D'Angelo A, Franco B: The dynamic cilium in human diseases. Pathogenetics 2009; 2: 3.

52 Louvi A, Grove EA: Cilia in the CNS: the quiet organelle claims center stage. Neuron 2011; 69: 1046-1060.

53 Basten SG, Giles RH: Functional aspects of primary cilia in signaling, cell cycle and tumorigenesis. Cilia 2013; 2: 6

54 Doherty D: Joubert syndrome: insights into brain development, cilium biology, and complex disease. Semin Pediatr Neurol 2009; 16: 143-154.

55 Ozonoff S, Williams BJ, Gale S, Miller JN: Autism and autistic behavior in Joubert syndrome. J Child Neurol 1999; 14: 636-641.

56 Artigas-Pallares J, Gabau-Vila E, Guitart-Feliubadalo M: (Syndromic autism: II. Genetic syndromes associated with autism) El autismo sindromico: II. Sindromes de base genetica asociados a autismo. Rev Neurol 2005; 40: S151-S162.

57 Holroyd S, Reiss AL, Bryan RN: Autistic features in Joubert syndrome: a genetic disorder with agenesis of the cerebellar vermis. Biol Psychiatry 1991; 29 287-294.

58 Alvarez Retuerto Al, Cantor RM, Gleeson JG et al: Association of common variants in the Joubert syndrome gene (AHI1) with autism. Hum Mol Genet 2008; 17: 3887-3896.

59 Wrana JL: Signaling by the TGFbeta superfamily. Cold Spring Harb Perspect Biol 2013; 5: a011197.

60 Okada K, Hashimoto K, Iwata Y et al: Decreased serum levels of transforming growth factor-beta1 in patients with autism. Prog Neuropsychopharmacol Biol Psychiatry 2007; 31: 187-190.

61 Ashwood P, Enstrom A, Krakowiak P et al: Decreased transforming growth factor beta1 in autism: a potential link between immune dysregulation and impairment in clinical behavioral outcomes. J Neuroimmunol 2008; 204: 149-153.

62 Vargas DL, Nascimbene C, Krishnan C, Zimmerman AW, Pardo CA: Neuroglial activation and neuroinflammation in the brain of patients with autism. Ann Neurol 2005; 57: 67-81.

63 McElhanon BO, McCracken C, Karpen S, Sharp WG: Gastrointestinal symptoms in autism spectrum disorder: a meta-analysis. Pediatrics 2014; 133: 872-883.

64 Coury DL, Ashwood P, Fasano A et al: Gastrointestinal conditions in children with autism spectrum disorder: developing a research agenda. Pediatrics 2012; 130: S160-S168.

65 Margolis KG, Li Z, Stevanovic K et al: Serotonin transporter variant drives preventable gastrointestinal abnormalities in development and function. J Clin Invest 2016; 126: 2221-2235

66 Kerin T, Ramanathan A, Rivas K, Grepo N, Coetzee GA, Campbell DB: A noncoding RNA antisense to moesin at 5p14.1 in autism. Sci Trans/ Med 2012; 4 128ra140r.

67 Almasy L: The role of phenotype in gene discovery in the whole genome sequencing era. Hum Genet 2012; 131: 1533-1540.

68 Geschwind DH: Advances in autism. Annu Rev Med 2009; 60: 367-380.

69 Armstrong K, larocci G: Brief report: the autism spectrum quotient has convergent validity with the social responsiveness scale in a high-functioning sample. J Autism Dev Disord 2013; 43: 2228-2232.

70 Kircher M, Witten DM, Jain P, O'Roak BJ, Cooper GM, Shendure J: A general framework for estimating the relative pathogenicity of human genetic variants. Nat Genet 2014; 46: 310-315.

Supplementary Information accompanies this paper on European Journal of Human Genetics website (http://www.nature.com/ejhg) 